\title{
Incidence of spontaneous notifications of adverse reactions with aceclofenac, meloxicam, and rofecoxib during the first year after marketing in the United Kingdom
}

\author{
Anna Raber ${ }^{\prime}$ \\ Joan Heras' \\ Joan Costa ${ }^{2}$ \\ Josep Fortea' \\ Albert Cobos ${ }^{3}$ \\ 'Medical Department, Almirall \\ Prodesfarma, Barcelona, Spain; \\ ${ }^{2}$ Department of Clinical \\ Pharmacology, Hospital Germans \\ Trias i Pujol, Barcelona, Spain; \\ ${ }^{3}$ Dept. of Public Health Bioestatistics \\ Unit. University of Barcelona, \\ Barcelona, Spain
}

\begin{abstract}
The objective was to compare the incidence of adverse reactions reported with three nonsteroidal anti-inflammatory drugs with different cyclo-oxygenase (COX)-2 selectivity. All spontaneous adverse reaction notifications in the pharmacovigilance database of the World Health Organisation Collaborating Centre for International Drug Monitoring with aceclofenac, meloxicam, and rofecoxib that were recorded during the first year of marketing were included. The incidence rate (adverse reactions $/ 10^{6}$ defined daily dose) and $95 \%$ confidence interval for total adverse reactions was 8.7 (6.1-12.0) for aceclofenac, 24.8 (23.1-26.6) for meloxicam, and 52.6 (49.9-55.4) for rofecoxib. Aceclofenac had a lower incidence of gastrointestinal bleeding, abdominal pain, and arterial hypertension than meloxicam and a lower incidence of gastrointestinal bleeding, abdominal pain, liver toxicity, thromboembolic cardiovascular events, arterial hypertension, and edema than rofecoxib. The incidence of total and gastrointestinal adverse reactions was significantly lower with aceclofenac than with meloxicam or rofecoxib, thus raising doubts about the hypothetical advantage of COX-2 selective inhibitors.
\end{abstract}

Keywords: pharmacovigilance, post-marketing, adverse reactions, nonsteroidal antiinflammatory drugs, aceclofenac, meloxicam, rofecoxib

\section{Introduction}

The treatment of inflammation and pain is an important area of therapeutics. In the last decade, nonsteroidal anti-inflammatory drugs (NSAIDs) have played a central role in these indications and they are currently considered the first choice, being one of the most widely prescribed drugs. However, their adverse reactions are important; gastrointestinal toxicity is the most frequent serious adverse effect, causing a considerable number of hospitalizations and deaths each year around the world (Simon 1995; Peloso 1996; Singh et al 1998; Wolfe et al 1999; Tramer et al 2000).

The effect of NSAIDs is mediated to a large extent by inhibition of prostaglandin synthesis through cyclo-oxygenase (COX). COX has two iso-enzymes in humans: COX-1 has a cytoprotective function in the gastric mucosa (Vane and Botting 1998) and COX-2 is detected in several tissues when an inflammatory reaction takes place. This has led to the assumption that the anti-inflammatory action of NSAIDs is due to COX-2 inhibition, whilst the gastric side effects are due to COX-1 inhibition (Vane 1971; Seibert et al 1995; Vane and Botting 1998).

Faced with the possibility of obtaining an anti-inflammatory effect without damaging the gastric mucosa, COX-2 selective NSAIDs such as celecoxib and rofecoxib were developed. Demonstration of this supposed advantage was based on two large safety studies, the CLASS (Celecoxib Long Term Arthritis Study) and the VIGOR (Vioxx Gastrointestinal Outcomes Research) studies (Bombardier et al 2000; Silverstein et al 2000). Results from these studies seemed to confirm that the risk of
Correspondence: Anna Raber

Medical Department, Almirall

Prodesfarma, General Mitre I5I, 08022

Barcelona, Spain

Tel +34932913485

Fax +3493291 2804

Email araber@almirall.es 
secondary gastrointestinal effects was lower with COX-2 selective NSAIDs than with classic NSAIDs. However, several studies published subsequently have questioned those results (Mukherjee et al 2001; Hrachavec and Mora 2001; Wright et al 2001; Pedros et al 2002; Laporte et al 2004).

The adverse reactions detected during clinical trials are not sufficient to completely characterize the safety profile of a product. The data obtained during the initial years after the introduction of a new drug to the market allow a better knowledge of its true safety profile (Caulin 2002; UK MCA 2004). The objective of this study was to compare the incidence of spontaneous reports of adverse reactions, recorded in a well established pharmacovigilance system, during the first year after the introduction of three NSAIDs with different COX-2 selectivity: aceclofenac, meloxicam, and rofecoxib.

\section{Methods}

We analysed all spontaneous reports of adverse reactions with the three NSAIDs (aceclofenac, meloxicam, and rofecoxib) that were recorded through the pharmacovigilance system in the United Kingdom (UK) during the first complete year after their introduction to the market. The UK was selected because, by the time the study was undertaken, all three drugs had been marketed for more than one year in this country and because of the reputation of its pharmacovigilance system. Rofecoxib was selected as the COX-2 selective NSAID because it was the first to be introduced and was the best known. Subsequently, it was decided to incorporate a preferential COX-2 NSAID as control. Meloxicam was selected as its date of introduction to the UK market was as close as possible to that of aceclofenac. According to International Marketing Services
(IMS) Dataview, the dates of introduction to the UK market were March 1996, September 1996, and May 1999 for aceclofenac, meloxicam, and rofecoxib, respectively.

Data on adverse reaction reports were obtained from the World Health Organisation (WHO) Adverse Drug Reaction database through the WHO Collaborating Centre for International Drug Monitoring (Uppsala, Sweden). These data comprise all notifications made directly by physicians to the Medicines Control Agency (MCA) through the yellow-card system, and does not include those coming from pharmaceutical companies.

The adverse reactions reported were coded using preferred terms (PTs) of the WHO Adverse Reaction Terminology dictionary (version 2004:4). The analysis was limited to total adverse reactions and those of the seven groups of conditions most commonly associated with NSAIDs: gastrointestinal bleeding, abdominal pain, liver toxicity, renal toxicity, oedema, arterial hypertension, and thromboembolic cardiovascular events. The PTs included in each of these groups are shown in Table 1.

The incidence of adverse reactions was computed by combining the number of notifications and the exposure during the corresponding time period. For each of the three selected drugs, the incidence rate (IR) of the seven types of adverse reaction considered was computed by dividing the number of adverse reactions reported by the number of defined daily doses (DDDs) consumed in the relevant time period (1997 for aceclofenac and meloxicam, and 2000 for rofecoxib) and multiplying by $10^{6}$ (so that rates are expressed as number of adverse reactions per million DDD). The number of DDDs consumed of the oral form of each drug (adopted exposure measure) was estimated from the sales figures in standard units obtained from the IMS Midas database and the DDD for each compound. The DDD

Table I WHO-ART preferred terms included in the groups assessed

\begin{tabular}{|c|c|}
\hline Group & WHO-ART preferred terms \\
\hline Gastrointestinal bleeding & $\begin{array}{l}\text { Duodenal ulcer hemorrhagic, duodenal ulcer perforated, gastric ulcer hemorrhagic, gastric ulcer } \\
\text { perforated, gastrointestinal hemorrhage, hematemesis, hemorrhage rectum, melaena, esophageal } \\
\text { ulceration hemorrhage, peptic ulcer perforated. }\end{array}$ \\
\hline Abdominal pain & Abdominal pain \\
\hline Liver toxicity & $\begin{array}{l}\text { Bilirubinemia, gamma-GT increased, hepatic function abnormal, hepatitis, hepatitis cholestatic, } \\
\text { hepatocellular damage, jaundice, SGPT increased. }\end{array}$ \\
\hline Renal toxicity & Nephrosis, oliguria, renal failure acute, renal function abnormal, urine flow decreased. \\
\hline Edema & $\begin{array}{l}\text { Angioedema, face edema, edema, edema mouth, edema peripheral, edema periorbital, papilloedema, } \\
\text { tongue edema. }\end{array}$ \\
\hline Arterial hypertension & Hypertension, hypertension aggravated. \\
\hline $\begin{array}{l}\text { Thromboembolic cardiovascular } \\
\text { events }\end{array}$ & $\begin{array}{l}\text { Angina pectoris aggravated, myocardial infarction, embolism arterial, cerebrovascular disorder, peripheral } \\
\text { ischemia, thrombophlebitis, thrombophlebitis deep, transient ischemic attack. }\end{array}$ \\
\hline
\end{tabular}

Abbreviations: ART, adverse reactions terminology; GT, glutamyl transferase; SGPT, serum glutamic pyruvic transaminase; WHO, World Health Organisation. 
established as the minimal daily dose indicated for maintenance treatment with each product was $200 \mathrm{mg} /$ day for aceclofenac, $15 \mathrm{mg} /$ day for meloxicam, and $25 \mathrm{mg} /$ day for rofecoxib (WHO 2004). However, it was decided to establish an arbitrary dose of $7.5 \mathrm{mg} /$ day (instead of $15 \mathrm{mg}$ / day) for meloxicam, and $12.5 \mathrm{mg} /$ day (instead of $25 \mathrm{mg}$ /day) for rofecoxib, since these are the most common dosages used in everyday practice. This decision necessitated duplication of the denominator used to compute the IR, so that it was halved. Consequently, this decision implies a conservative estimate of the IR with meloxicam and rofecoxib.

The $95 \%$ confidence intervals (CIs) for the IRs were computed using the exact method based on the Poisson distribution implemented in the CIA software (BMJ Publishing, London, UK) (Altman et al 2000). To compare the IRs of two drugs, the IR ratio (IRR) was computed as $I R R=I R_{A} / I R_{C}$, where $I R_{A}$ is the point estimate of the IR with aceclofenac and $I_{C}$ is the point estimate of the IR with the comparator drug (meloxicam or rofecoxib). The 95\% CIs for the IRRs were computed using an exact method based on the Poisson distribution 21 implemented in a SAS ${ }^{\circledR}$ (v8.00) program (SAS Institute, Inc., Cary, NC, USA). The functions used in this SAS program were validated by reproducing example 9.2 from the book by Sahai and Khurshid (1996).

Due to the possibility of a bias resulting from secular trends affecting spontaneous reporting of adverse reactions in the period considered (from 1997, for aceclofenac and meloxicam, to 2000 for rofecoxib), we looked at the yearly global report rate during this period. Since the number of adverse reaction notifications showed a progressive increase in the period from 1996 to 2000, an adjustment was made to mitigate possible bias. To this end, we re-computed the IR of rofecoxib (and the IRR of aceclofenac vs rofecoxib) adjusting the rates for rofecoxib, to compensate for the relative over-reporting in 2000 with respect to 1997, as follows: adjusted IR $=$ IR $\times 0.82$. The correction factor of 0.82 was obtained by dividing the total number of adverse reaction reports in 1997 (174 543) by the corresponding number in 2000 (213 206).

\section{Results}

In the WHO database, a total of 36 adverse reactions were recorded with aceclofenac and 769 with meloxicam during 1997, and 1743 with rofecoxib during 2000.

During 1997, the number of units sold in the UK was 138400 for aceclofenac (PRESERVEX, 60 tablets $100 \mathrm{mg}$ ) and 867300 for meloxicam (MOBIC, 30 tablets $7.5 \mathrm{mg} / 30$ tablets $15 \mathrm{mg} / 12$ suppositories $15 \mathrm{mg}$ ). In 2000, 592000 units of rofecoxib (VIOXX, 28 tablets $12.5 \mathrm{mg} / 28$ tablets $25 \mathrm{mg}$ ) were sold. These consumption data imply an exposure of 4152000 DDD for aceclofenac, 30963000 DDD for meloxicam and 27104000 DDD for rofecoxib.

The IRs $(95 \% \mathrm{CI})$ of all adverse reactions per million DDD were 8.7 (6.1-12.0) for aceclofenac, 24.8 (23.1-26.6) for meloxicam and $52.6(49.9-55.4)$ for rofecoxib. The IRs of adverse reactions for each of the seven groups considered are shown in Table 2. In general, the IRs with aceclofenac were lower than those with meloxicam or rofecoxib for gastrointestinal events and hypertension, whilst those for liver and renal toxicity, thromboembolic events and oedema were lower with meloxicam than with aceclofenac. In all cases except renal toxicity, the highest incidence was observed with rofecoxib, even after adjustment for the

Table 2 Incidence rates (95\% confidence interval [Cl]) of spontaneous reports of adverse reactions with aceclofenac, meloxicam, and rofecoxib during the first year after introduction to the UK market

\begin{tabular}{|c|c|c|}
\hline $\begin{array}{l}\text { Adverse reaction } \\
\text { group }\end{array}$ & $\begin{array}{l}\text { Incidence rate } \\
(95 \% \mathrm{Cl})\end{array}$ & $\begin{array}{l}\text { Adjusted } \\
\text { incidence rate } \\
(95 \% \mathrm{Cl})\end{array}$ \\
\hline \multicolumn{3}{|c|}{ Gastrointestinal bleeding } \\
\hline Aceclofenac & $0.000(0.000-0.888)$ & \\
\hline Meloxicam & $1.421(1.033-1.908)$ & \\
\hline Rofecoxib & $3.247(2.604-4.000)$ & $2.656(2.078-3.345)$ \\
\hline \multicolumn{3}{|l|}{ Abdominal pain } \\
\hline Aceclofenac & $0.000(0.000-0.888)$ & \\
\hline Meloxicam & $1.356(0.978-1.833)$ & \\
\hline Rofecoxib & $3.837(3.135-4.649)$ & $3.136(2.505-3.878)$ \\
\hline \multicolumn{3}{|l|}{ Livertoxicity } \\
\hline Aceclofenac & $0.24 I(0.006-1.342)$ & \\
\hline Meloxicam & $0.032(0.00 I-0.180)$ & \\
\hline Rofecoxib & $0.775(0.480-1.184)$ & $0.627(0.365-1.004)$ \\
\hline \multicolumn{3}{|l|}{ Renal toxicity } \\
\hline Aceclofenac & $0.723(0.149-2.112)$ & \\
\hline Meloxicam & $0.097(0.020-0.283)$ & \\
\hline Rofecoxib & $0.627(0.365-1.004)$ & $0.5 \mathrm{I} 7(0.282-0.867)$ \\
\hline \multicolumn{3}{|c|}{ Thromboembolic cardiovascular events } \\
\hline Aceclofenac & $0.24 \mid(0.006-1.342)$ & \\
\hline Meloxicam & $0.065(0.008-0.233)$ & \\
\hline Rofecoxib & $0.664(0.394-1.050)$ & $0.553(0.310-0.913)$ \\
\hline \multicolumn{3}{|l|}{ Arterial hypertension } \\
\hline Aceclofenac & $0.000(0.000-0.888)$ & \\
\hline Meloxicam & $0.097(0.020-0.283)$ & \\
\hline Rofecoxib & 0.701 (0.422-1.095) & $0.590(0.337-0.959)$ \\
\hline \multicolumn{3}{|l|}{ Edema } \\
\hline Aceclofenac & I.204 (0.39I-2.8I0) & \\
\hline Meloxicam & I.I $30(0.787-1.572)$ & \\
\hline Rofecoxib & $4.833(4.04 I-5.735)$ & $3.948(3.235-4.77)$ \\
\hline
\end{tabular}


positive secular trend in the overall number of adverse reaction reports.

The IRRs of aceclofenac versus meloxicam and of aceclofenac versus rofecoxib are displayed in Table 3. A CI excluding unity reflects a significant difference in the IR between the two drugs being compared. In the case of gastrointestinal bleeding and abdominal pain, the IR was significantly lower with aceclofenac than with meloxicam or rofecoxib. In the case of edema, the IR with aceclofenac was significantly lower than with rofecoxib. In the remaining cases, the width of the CI does not allow conclusive differences to be established.

\section{Discussion}

The data in this study are based on spontaneous reports of adverse reactions recorded through the pharmacovigilance system in the UK. This allows for a fair comparative assessment of post-marketing safety information since the same notification system is used for all three drugs. The UK was selected because all three NSAIDs were already marketed, the times at which they were introduced to the market were relatively close, and finally because of the good reputation of the UK yellow-card pharmacovigilance system.

Table 3 Incidence rate ratios (95\% confidence intervals [Cl]) of adverse reactions with aceclofenac versus meloxicam, and with aceclofenac versus rofecoxib

\begin{tabular}{|c|c|c|}
\hline $\begin{array}{l}\text { Adverse reaction } \\
\text { group }\end{array}$ & $\begin{array}{l}\text { Incidence rate } \\
\text { ratio }(95 \% \mathrm{Cl})\end{array}$ & $\begin{array}{l}\text { Adjusted } \\
\text { incidence rate } \\
\text { ratio }(95 \% \mathrm{Cl})\end{array}$ \\
\hline \multicolumn{3}{|c|}{ Gastrointestinal bleeding } \\
\hline Aceclofenac/meloxicam & $0.00(0.00-0.65)$ & \\
\hline Aceclofenac/Rofecoxib & $0.00(0.00-0.28)$ & $0.00(0.00-0.34)$ \\
\hline \multicolumn{3}{|l|}{ Abdominal pain } \\
\hline Aceclofenac/meloxicam & $0.00(0.00-0.68)$ & \\
\hline Aceclofenac/rofecoxib & $0.00(0.00-0.24)$ & $0.00(0.00-0.29)$ \\
\hline \multicolumn{3}{|l|}{ Livertoxicity } \\
\hline Aceclofenac/meloxicam & $7.46(0.10-585.39)$ & \\
\hline Aceclofenac/rofecoxib & $0.31(0.01-1.93)$ & $0.38(0.0 \mathrm{I}-2.45)$ \\
\hline \multicolumn{3}{|l|}{ Renal toxicity } \\
\hline Aceclofenac/meloxicam & $7.46(1.00-55.68)$ & \\
\hline Aceclofenac/rofecoxib & $1.15(0.22-3.98)$ & $1.40(0.26-5.01)$ \\
\hline \multicolumn{3}{|c|}{ Thromboembolic cardiovascular events } \\
\hline Aceclofenac/meloxicam & $3.73(0.06-71.62)$ & \\
\hline Aceclofenac/rofecoxib & $0.36(0.01-2.30)$ & $0.43(0.01-2.83)$ \\
\hline \multicolumn{3}{|l|}{ Arterial hypertension } \\
\hline Aceclofenac/meloxicam & $0.00(0.00-18.05)$ & \\
\hline Aceclofenac/Rofecoxib & $0.00(0.00-1.40)$ & $0.00(0.00-1.69)$ \\
\hline \multicolumn{3}{|l|}{ Edema } \\
\hline Aceclofenac/meloxicam & $1.07(0.33-2.73)$ & \\
\hline Aceclofenac/rofecoxib & $0.25(0.08-0.60)$ & $0.31(0.10-0.73)$ \\
\hline
\end{tabular}

Although pharmacovigilance is an essential process to establish the safety of a drug and to detect rare adverse reactions, the analysis of pharmacovigilance data has important limitations. Firstly, the information is not always homogeneous, at least in terms of the source (which is not always available). Secondly, under-reporting is very common. Finally, the difficulty in establishing a causal relationship between the adverse reaction and the drug administered is possibly the most serious limitation. It is important to bear in mind that, in the case of spontaneous reports, much of the data needed for a causality assessment, including chronology and the possible contribution of concomitant medications and diseases, are not always available. For these reasons, the WHO states that studies such as the one presented here do not necessarily reflect their opinion. In any case, these limitations are inherent to the pharmacovigilance system itself, and cannot be overcome when data from such systems are analyzed.

When pharmacovigilance data are used, as in the current study, to compare the safety profiles of different drugs, it is important to take into account that the frequency of notifications is dependent on the time elapsed since the introduction to the market, typically reaching a maximum by the end of the second year and subsequently decreasing. To avoid a biased comparison due to this time evolution pattern, the first year after the introduction of each drug was studied. It is also important to adjust for possible secular trends in the reporting, as we did for the adverse reactions reported with rofecoxib during 2000.

The number of notifications with a product may be influenced by advertising and may depend on the expectation and degree of awareness of physicians and other health professionals (Malhotra et al 2004). Unfortunately, there is no simple and reliable way to measure these factors so that they may be taken into account.

This study shows that the incidence of spontaneous reports of adverse reactions with aceclofenac is, in general, lower than it is with the comparators, particularly rofecoxib. This is surprising since rofecoxib is a selective COX-2 inhibitor and, consequently, a better gastrointestinal safety profile might be expected. Although, presumably, there could be a trend for rofecoxib to be prescribed for patients with a higher gastrointestinal risk, our findings do not confirm the hypothetical advantage for rofecoxib at the gastrointestinal level, but instead suggest the possibility that its safety profile is still inferior to that of aceclofenac. 
The results of the study suggest that aceclofenac may have a significantly lower risk of gastrointestinal bleeding, abdominal pain and oedema than meloxicam or rofecoxib.

Due to the nature and source of the data used, there is no guarantee that the results reflect the real incidence of adverse reactions, and therefore it is not possible to definitively conclude that aceclofenac has a lower incidence of gastrointestinal bleeding or abdominal pain than meloxicam or rofecoxib. For instance, the expectation gained from previous results that rofecoxib has a better gastrointestinal safety profile might have induced a bias if it was preferentially indicated in high risk patients ("channeling bias") (Wolfe, Flowers, et al 2002; MacDonald et al 2003) or if proton-pump inhibitors were less frequently used in association. Thus, in a study conducted in the UK that compared two cohorts of 1127 and 1376 patients treated with rofecoxib and meloxicam, respectively, gastrointestinal antecedents were more common in the cohort treated with rofecoxib, but the IRs of upper gastrointestinal complications were not significantly different after adjustment for this risk factor (Layton et al 2003). However, in a recent study conducted in 18 hospitals in Spain and Italy that analyzed 2813 cases and 7193 controls, neither the risk profile nor the use of gastric protectors differed between patients treated with rofecoxib and those given aceclofenac (Laporte et al 2004). Therefore, a selection bias does not always appear to be present, although the number of patients exposed to the newer NSAID was small. Other studies have reported the existence of possible channeling bias for GI effects or use of gastroprotective agents (Wolfe, Flowers, et al 2002; Moride et al 2005), severity of illness and cardiovascular risk (paradoxically patients at higher risk were channeled to COX-2 inhibitors) (Zhao et al 2002; Rawson et al 2005).

In a study that analyzed 106564 patients starting treatment with a NSAID, proton pump inhibitors were more frequently given with COX-2 inhibitors than with traditional NSAIDs (Ofman et al 2004). However, in a similar study based on data from 69648 patients, the use of proton pump inhibitors was less common in patients treated with COX-2 inhibitors that in those treated with traditional NSAIDs. Unfortunately, we cannot know whether or not this phenomenon affected our data or, if so, how it was affected. Finally, the previously mentioned study of cases and controls reported estimates of gastrointestinal bleeding risk of 1.4 per million cases for aceclofenac, 5.7 for meloxicam, and 7.2 for rofecoxib (Laporte et al 2002). This safety profile pattern is comparable with the one observed in our study.
In summary, we would like to draw attention to the usefulness of the safety alert system. Recently, we have seen the unexpected withdrawal of Vioxx ${ }^{\circledR}$ based on an unforeseen cardiovascular risk (Merck 2004). This fact has triggered the risk/benefit re-evaluation of this therapeutic class by the main regulatory agencies (Okie 2005).

The use of this type of pharmacovigilance raw data analysis might contribute to early characterization of the safety profile knowledge of such drugs.

\section{Conclusions}

Spontaneous reports of adverse reactions recorded during the first year after introduction of aceclofenac, meloxicam, and rofecoxib in the UK revealed IRs of total adverse reactions, gastrointestinal bleeding, abdominal pain, and edema that are notably lower for aceclofenac than for meloxicam or rofecoxib. Although the nature of the data does not guarantee that these results reflect a real lower incidence of these reactions with aceclofenac, the magnitude of the differences observed provide a clear indication that the hypothetical advantage of COX-2 selective inhibitors may not translate into clinical safety advantages regarding gastrointestinal bleeding or overall safety.

\section{Acknowledgements}

This work was supported by Almirall Prodesfarma. RDES, SL was contracted to perform the statistical analysis and prepare the manuscript. We are grateful to Dr Salvador Bergoñón (RDES, SL) for his comments on the manuscript that contributed significantly to its improvement.

\section{References}

Altman DG, Machin D, Bryant TN, et al (eds). 2000. Statistics with Confidence. Bristol: BMJ Bks.

Bombardier C, Laine L, Reicin A, et al. 2000. Comparison of upper gastrointestinal toxicity of rofecoxib and naproxen in patients with rheumatoid arthritis. VIGOR Study Group. N Engl J Med, 343:1520-8.

Caulin C. 2002. From the chemical product to the authorization of drug marketing. Rev Prat, 52:486-90.

Hrachavec JB, Mora M. 2001. Reporting of 6-month vs 12-month data in a clinical trial of celecoxib. Author reply. JAMA, 286:2399-400.

Laporte JR, Ibanez L, Vidal X, et al. 2004. Upper gastrointestinal bleeding associated with the use of NSAIDs: newer versus older agents. Drug Saf, 27:411-20.

Layton D, Heeley E, Hugues K, et al. 2003. Comparison of the incidence rates of selected gastrointestinal events reported for patients prescribed rofecoxib and meloxicam in general practice in England using prescription-event monitoring data. Rheumatology, 42:622-31.

MacDonald TM, Morant SV, Goldstein JL, et al. 2003. Channelling bias and the incidence of gastrointestinal haemorrhage in users of meloxicam, coxibs, and older, non-specific non-steroidal antiinflammatory drugs. Gut, 52:1265-70.

Malhotra S, Pandhi P, Shafiq N. 2004. COX-2 inhibitors: a CLASS act or Just VIGORously promoted. MedGenMed, 6:6. 
Merck \& Co. 2004. Merck anuncia la retirada voluntaria de la venta de VIOXX® en todo el mundo [online]. Accessed on 3 October 2004. URL: http://www.vioxx.com/vioxx/documents/spanish/ vioxx_press_release.pdf.

Moride Y, Ducruet T, Boivin JF, et al. 2005. Prescription channeling of COX-2 inhibitors and traditional nonselective nonsteroidal antiinflammatory drugs: a population-based case-control study. Arthritis Res Ther, 7:R333-42.

Mukherjee D, Nissen SE, Topol EJ. 2001. Risk of cardiovascular events associated with selective COX-2 inhibitors. JAMA, 286:954-9.

Ofman JJ, Badamgaray E, Henning JM, et al. 2004. Utilization of nonsteroidal anti-inflammatory drugs and antisecretory agents: a managed care claims analysis. Am J Med, 116:835-42.

Okie S. 2005. Raising the safety bar - the FDA's coxib meeting. $N$ Eng $J$ Med, 352:13.

Pedros C, Cerecza G, Laporte JR. 2002. Primeras notificaciones espontáneas de sospechas de reacciones adversas a los nuevos antiinflamatorios selectivos sobre la ciclooxigenasa-2. Med Clin, 118:415-17.

Peloso PM. 1996. Strategies and practice for use of nonsteroidal antiinflammatory drugs. Scand J Rheumatol, 105(Supp1):29-43.

Rawson NS, Nourjah P, Grosser SC, et al. 2005. Factors associated with celecoxib and rofecoxib utilization. Ann Pharmacother, 39:597-602.

Sahai H, Khurshid A. 1996. Statistics in epidemiology. methods, techniques and applications. Boca Raton: CRC Pr.

Seibert K, Masferrer J, Zhang Y, et al. 1995. Mediation of inflammation by cyclooxygenase-2. Agents Actions, 46(Suppl):41-50.

Silverstein FE, Faich G, Goldstein JL, et al. 2000. Gastrointestinal toxicity with celecoxib vs nonsteroidal anti-inflammatory drugs for osteoarthritis and rheumatoid arthritis: the CLASS study: a randomized controlled trial. Celecoxib Long-term Arthritis Safety Study. JAMA, 284:1247-55.

Simon LS. 1995. Actions and toxicity of nonsteroidal anti-inflammatory drugs. Curr Opin Rheumatol, 7:159-66.
Singh G, Rosen Ramey DR. 1998. NSAID reduced gastrointestinal complications: the ARAMIS perspective - 1997. Arthritis, Rheumatism and Aging Medical Information System. J Rheumatol, 51(Suppl):816.

Tramer MR, Moore RA, Reynolds DJ, et al. 2000. Quantitative estimation of rare adverse events which follow a biological progression: a new model applied to chronic NSAID use. Pain, 85:169-82.

[UK MCA] Medicines and Healthcare products Regulatory Agency. 2004. Monitoring the safety and quality of medicines: Pharmacovigilance [online]. Accessed on 11 August 2004. URL: http://www.mhra.gov.uk.

Vane JR, Botting RM. 1998. Mechanism of action of nonsteroidal antiinflammatory drugs. Am J Med, 104:2S-8S.

Vane JR. 1971. Inhibition of prostaglandin synthesis as a mechanism of action for aspirin-like drugs. Nature, 231:232-5.

[WHO] Norwegian Institute of Public Health. 2004. World Health Organisation Collaborating Centre for Drug Statistics Methodology [online]. Accessed on 3 October 2004. URL: http://www.whocc.no/ atcddd.

Wolfe F, Anderson J, Burke TA, et al. 2002. Gastroprotective therapy and risk of gastrointestinal ulcers: risk reduction by COX-2 therapy. $J$ Rheumatol, 29:467-73.

Wolfe F, Flowers N, Burke TA, et al. 2002. Increase in lifetime adverse drug reactions, service utilization, and disease severity among patients who will start COX-2 specific inhibitors: quantitative assessment of channeling bias and confounding by indication in 6689 patients with rheumatoid arthritis and osteoarthritis. J Rheumatol, 29:1015-22.

Wolfe MM, Lichtenstein DR, Singh G. 1999. Gastrointestinal toxicity of nonsteroidal anti-inflammatory drugs. $N$ Engl J Med, 340:1888-99.

Wright JM, Perry TL, Bassett KL, et al. 2001. Reporting of 6-month vs 12-month data in a clinical trial of celecoxib. JAMA, 286:2398-400.

Zhao SZ, Burke TA, Whelton A, et al. 2002. Comparison of the baseline cardiovascular risk profile among hypertensive patients prescribed COX-2-specific inhibitors or nonspecific NSAIDs: data from reallife practice. Am J Manag Care, 8(15 Suppl):S392-400. 